\title{
Micro-Nano-Pico-satellites: Complementary Tools for Big Astrophysics
}

\author{
René Hudec ${ }^{1,2 * \dagger}$ \\ ${ }^{1}$ Czech Technical University in Prague, Faculty of Electrical Engineering, \\ Technicka 2, CZ 16000 Prague, Czech Republic \\ ${ }^{2}$ Kazan Federal University, Kazan, Russian Federation \\ E-mail: rene.hudec@gmail.com
}

The picosatellites, recently in development at many institutes and universities worldwide (e.g. Schmidt et al. 2009 and Schilling 2006), represent, apart from growing commercial applications, a challenge for space astronomy and astrophysics, as they can act as complementary tools for modern space astrophysics.

Frontier Research in Astrophysics - III (FRAPWS2018)

28 May - 2 June 2018

Mondello (Palermo), Italy

\footnotetext{
* Speaker.

${ }^{\dagger}$ A footnote may follow.
} 


\section{Introduction}

The miniature satellites are recently in development at many institutes and universities, in many cases with a participation of students. The fast development of the related techniques and technologies enables the growing commercial applications, but also to consider scientific applications of these small satellites. Below we give a small summary of pico (cube) and nanosatellites. The CubeSat standard size is 1 Liter Volume, i.e. $10 \times 10 \times 10 \mathrm{~cm}$, and a typical weight is $1.3 \mathrm{~kg}$. Multiple modules are possible, i.e. $3 \mathrm{U}=3$ modules/units, i.e. $10 \times 10 \times 30 \mathrm{~cm}$, typically up to 12U. The typical masses are as follows: femtosatellite -10 to $100 \mathrm{~g}$, picosatellite -0.1 to $1 \mathrm{~kg}$, nanosatellite -1 to $10 \mathrm{~kg}$, microsatellite -10 to $100 \mathrm{~kg}$

\section{Pico and nanosatellites for astrophysics}

Typically, the picosatellite missions represent a cost effective solution for space astronomy and astrophysics and also less strict requirements for space qualification of used components if compared to larger satellites developed by space agencies. However, there are still the following strict requirements for the scientific payload for picosatellites. (i) The scientific payload must fit into a small volume, typically $30 \times 10 \times 10 \mathrm{~cm}$ or less (3U, i.e. 3 cubesat modules), (ii) it must be of low weight, less than $1 \mathrm{~kg}$, (iii) with low power consumption of about 10 Watts or less.

Due to above mentioned limitations, it is, in general, not easy to find suitable scientific payload still able to provide valuable scientific measurements.

In addition to scientific goals, the picosatellite missions can be used for technological tests such as TRW (Technology Readiness Level, a method for estimating the maturity of technologies during the acquisition phase of a program, developed at NASA during the 1970s and nowadays used by space agencies including ESA) increase, flight demonstrations, etc.
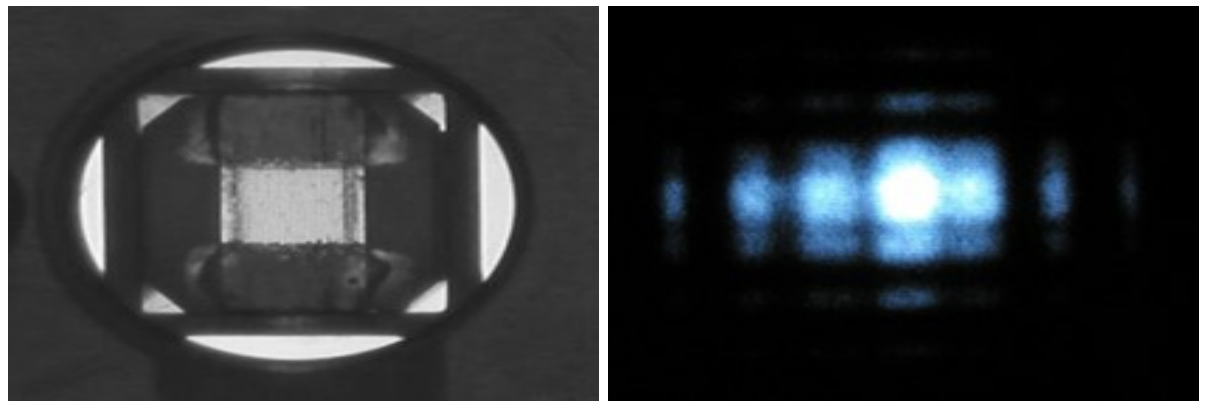

Figure 1: Micro Lobster Eye (LE) X-ray module: 3 x 3 x 14 mm module based on glass foils 30 microns thick separated by 70 microns (left) and a measured focal image of distant point-like source at $8 \mathrm{keV} \mathrm{X}$-rays from the micro LE module (right).

The goal of this paper is to give and discuss ideas for picosatellites astrophysical applications, i.e. scientific payloads. It is not describing any particular space mission hence no details about the real space mission such as processing resources, ground support system and TTC (Telemetry, Telecommand and Control), orbit, temperature requirements of detectors and their qualification plan, etc., are addressed here. 


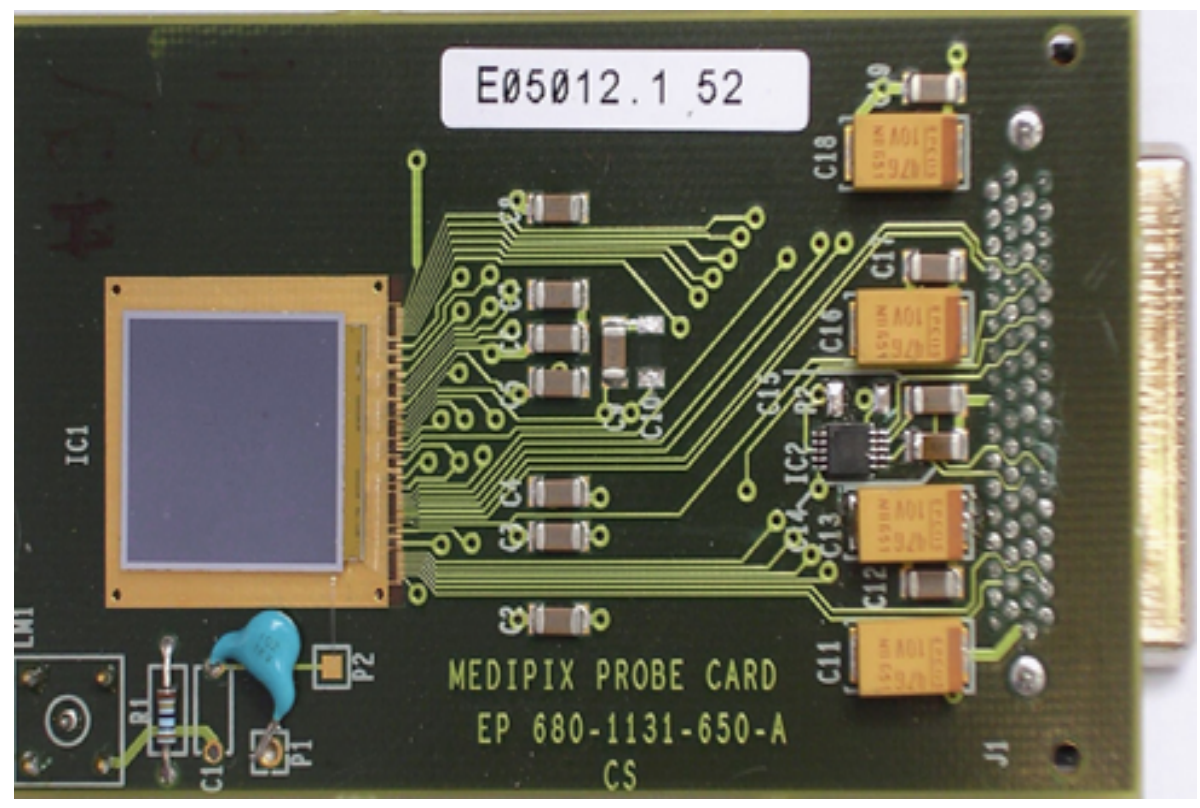

Figure 2: The Medipix focal detector board.
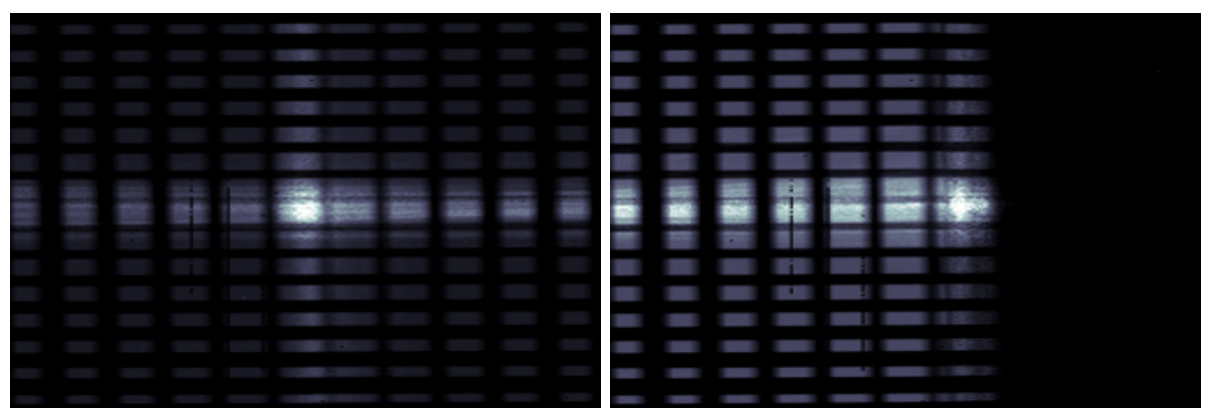

Figure 3: The X-ray image of miniature LE X-ray module recorded by the Medipix detector on axis (left) and off-axis (right).

\section{Miniature payloads for high-energy astrophysics}

Some astrophysical X-ray instrumentation can be succesfully miniaturized for cubesat applications. The most promising application area is represented by wide field X-ray lenses. The Lobster Eye (LE) X-ray optics was originally proposed by Schmidt (1975) and Angel (1979). Since then, numerous test specimens of Lobster Eye telescopes were designed and tested (e.g. Inneman et al. 1999; Hudec et al. 2000, 2003, 2004; Tichý et al. 2009, 2011). The Lobster-Eye (LE) X-ray telescope can be miniaturized for an application in picosatellites. The LE telescopes are novel wide field X-ray telescopes with the field of view (FOV) of 100 sq. deg. They are more easily possible (a classical X-ray optics has the FOV of only 1 deg or less) and are based on a real analogy with the lobster eyes.

The miniature $\mathrm{X}$-ray telescopes requires miniaturized focal detectors as well. The one of best 


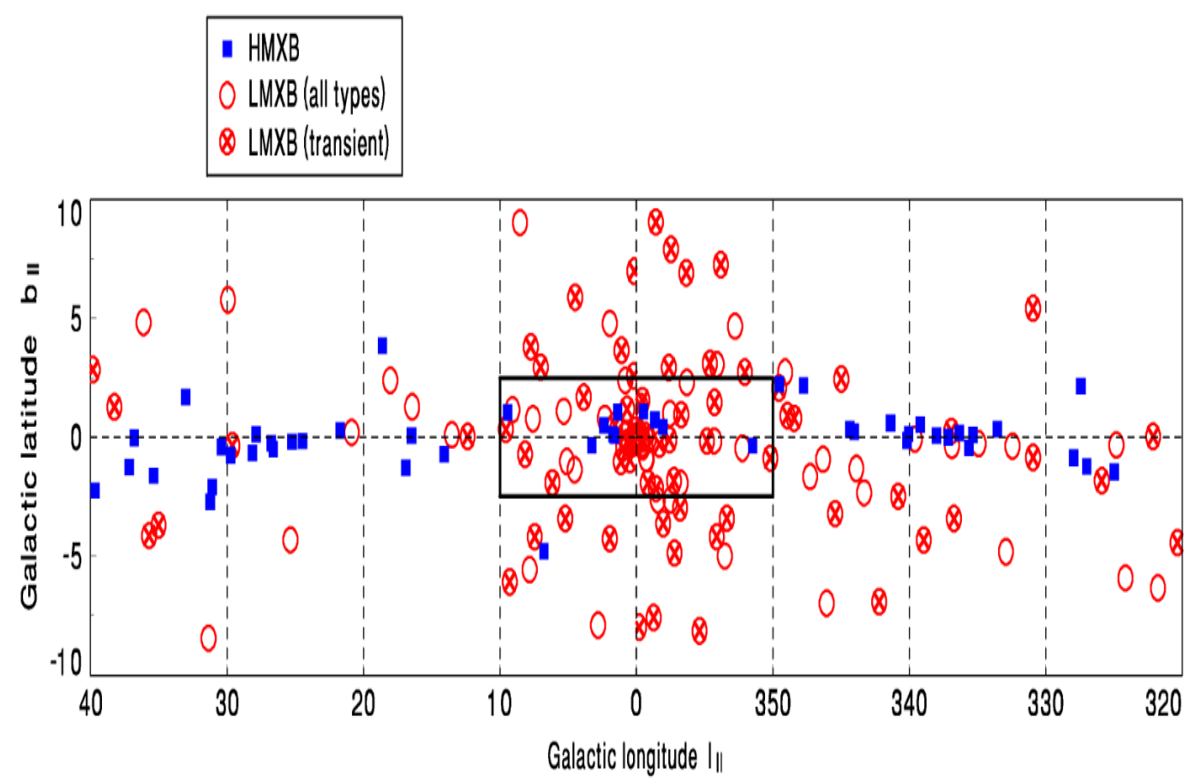

Figure 4: The field of the center of the Galaxy $(20$ x $80 \mathrm{deg})$. The positions of known LMXBs and HMXBs are marked. The field proposed for the monitoring by lobster is marked by the oblong - it contains a number of the already known objects.

available options is the detector Medipix (Timepix). Medipix is a family of photon counting pixel detectors developed by an international collaboration, hosted by CERN. The CTU in Prague is a member of this cooperation. The Medipix detector represents a suitable imaging detector for a use in space LE telescopes, as it is a pixelated photon counting semiconductor detector which features adjustable energy thresholds allowing multispectral X-ray imaging. These detectors offer several different working modes for X-ray imaging applications (e.g. Procz et al., 2012): (i) Single pixel mode (SPM): one threshold with a large 24-bit counter providing a high dynamic range or two thresholds with separate 12-bit counters providing two energy channels. This enables dual channel $\mathrm{X}$-ray imaging with a single acquisition, and (ii) Charge summing mode (CSM): to reduce the influence of charge sharing effects, in this mode charge deposited to adjacent pixels will be summed up and assigned to the pixel featuring the highest signal. The space application was demonstrated recently e.g. onboard the VZLUSAT1 cubesatellite (Baca et al., 2018).

The LE Telescope for a picosatellite can be demonstrated e.g. by the following example: Energy of $4.5 \mathrm{keV}=7.2 \times 10^{-6} \mathrm{erg}$, Focal length $250 \mathrm{~mm}, \mathrm{~h}=30 \mathrm{~mm}$, Weight less than $1 \mathrm{~kg}$ (optics: $50 \mathrm{~g}$, detector: $50 \mathrm{~g}$ ), FOV $2 \mathrm{deg} \times 2 \mathrm{deg}$, gain $=820$. Such device can provide daily minimal flux: $9.2 \times 10^{-10} \mathrm{erg} \mathrm{s}^{1} \mathrm{~cm}^{-2}$.

However, a more suitable spacecraft is a bit larger larger, e.g. 6U CubeSat as it allows to accommodate several LE modules to increase the final FOV (Tichý et al. 2013a). We elaborated a design of a new SLE prototype as follows. More mirror plates: 333 per set, a larger input area $(10 \times 10 \mathrm{~cm})$ and hence higher gain, a special coating to increase the reflectivity at higher energies, a better manufacturing technology, the focal length kept at $250 \mathrm{~mm}$, larger FOV (approximately $10 \times 10$ degrees) . 
Additional possibilities are provided by more advanced solutions such as cubesatellite tandem flights and fleets.

Wide field X-ray monitors of Lobster Eye type were demonstrated to play an important role in modern astrophysics (e.g. Hudec et al. 2007; Švéda et al. 2004). The most important scientific cases are briefly summarized below. (i) A long-term (months) measurement of the light curves of bright persistent X-ray binaries in the direction toward the center of the Galaxy in the soft X-ray band and (ii) Detection and measurement of the light curves of bright transient events of X-ray binaries in the direction toward the center of the Galaxy in the soft X-ray band.

The LE telescopes can typically serve in two basic operation modes as follows. (i) Starrying (pointed) mode - only for satellite with pointing, and (ii) Scanning mode (no satellite pointing and/or stabilization required).

The LE application on cubesat was demonstared on the VZLUSAT minisatellite (Urban et al., 2017, Baca et al., 2016). The spectral energy range can be extended toward higher energies up to $30 \mathrm{keV}$ aby application of 1D (one dimensional) LE arrangement (Pina et al., 2016). This is based on the fact that there is only one reflection in 1D systems, compared to the two reflections in the full LE (2D) system..

\section{Conclusions}

The recent and future picosatellites with extended lifetime offer excellent platforms for cost effective astrophysical payloads providing reasonable scientific results. The proposed mission (miniature LE X-ray telescope/monitor in a picosatellite) can acquire scientifically important data for a low price, if compared to large satellite missions. The optics for the presented mission is feasible and already demonstrated. The scientific justification is strong, including several perspective areas of modern astrophysics. A new prototype of the optics (based on the optimized design for a picosatellite) is to be developed and tested.

Acknowledgments This work was supported by the grant GA CR 13-33324S. We also acknowledge the project AHEAD funded by the European Union as Research and Innovation Action under Grant No: 654215.

\section{References}

[1] Hudec, R., et al., Proc. SPIE 5488, UV and Gamma-Ray Space Telescope Systems, (11 October 2004); doi: 10.1117/12.551915, 2004

[2] Hudec, R., Pína, L., Inneman, A., Švéda, L., LOBSTER - Astrophysics with Lobster Eye Telescopes, in Exploring the Cosmic Frontier, ESO Astrophysics Symposia European Southern Observatory 2007, pp.73-74, 2007

[3] Angel, J. R. P., 1979, Astroph. J., 364, 233

[4] Inneman, A., et al., 2000, Proc. SPIE, 4138, 94

[5] Hudec, R., et al., 2000, SPIE Proc. 4012, 432

[6] Hudec, R., et al., 2003, SPIE Proc. 4851, 578

[7] Hudec, R., et al., 2004a, SPIE Proc. 5488, 449 
[8] Hudec, R., et al., 2004b, Nucl. Phys. B Proc. Suppl. 132, 320

[9] Procz, S., et al., Medipix 3 CT for material sciences Proc. 14th INTERNATIONAL WORKSHOP ON RADIATION IMAGING DETECTORS, 1âĂŞ5 JULY 2012, FIGUEIRA DA FOZ, PORTUGAL, PUBLISHED BY IOP PUBLISHING FOR SISSA MEDIALAB, 2012. doi:10.1088/1748-0221/8/01/C01025

[10] Schilling, K., Design of Pico-Satellites for Education in Systems Engineering. In: IEEE Aerospace and Electronic Systems Magazine 21 (2006), S. 9-14, 2006

[11] Schmidt, M., Ravandoor, K., Kurz, O., Busch, S., Schilling, K., Attitude Determination for the Pico-Satellite UWE-2. In: Space Technology 28. 2009, pp.67-74, 2009

[12] Schmidt, W. K. H., 1975, NucIM, 127, 285

[13] Švéda, L., et al., 2004, SPIE Proc. 5168, 393

[14] Tichý, V., et al., 2009, Balt. Astr. 18, 362

[15] Tichý, V., et al., 2011, Nucl. Instr. Meth. A, A633, S169

[16] Tichý, V., et al., 2013a, SPIE Proc. 8777, 877711

[17] Tichý, V., 2013b, SPIE Proc. 8777, 877710

[18] Baca, T., M. Jilek, I. Vertat, M. Urban, O. Nentvich, R. Filgas, C. Granja, A. Inneman, and V. Daniel: Timepix in LEO Orbit onboard the VZLUSAT-1 Nanosatellite: 1-year of Space Radiation Dosimetry Measurements, Journal of Instrumentation, Volume 13, id.C11010 (2018).

[19] Urban, M., O. Nentvich, V. Stehlikova, T. Baca, V. Daniel, and R. Hudec: VZLUSAT-1: Nanosatellite with miniature lobster eye X-ray telescope and qualification of the radiation shielding composite for space application, Acta Astronautica, Volume 140, id.96 (2017)

[20] Baca, T., M. Platkevic, J. Jakubek, A. Inneman, V. Stehlikova, M. Urban, O. Nentvich, M. Blazek, R. McEntaffer, and V. Daniel: Miniaturized X-ray telescope for VZLUSAT-1 nanosatellite with Timepix detector, Journal of Instrumentation, Volume 11, id.C10007 (2016)

[21] Daniel, V., L. Pina, A. Inneman, et al.,: Terrestrial gamma-ray flashes monitor demonstrator on CubeSat, CubeSats and NanoSats for Remote Sensing, Volume 9978, id.99780D (2016)

[22] Pina, L., R. Hudec, A. J. Inneman, T. Baca, M. Blazek, M. Platkevic, L. Sieger, D. Doubravova, R. L. McEntaffer, T. B. Schultz, and V. Daniel: Development and tests of x-ray multifoil optical system for 1D imaging (Conference Presentation), Advances in Laboratory-based X-Ray Sources, Optics, and Applications V, Volume 9964, id.99640B (2016) 